\title{
A SIMPLE FORMULATION OF LIP BALM USING CARROT EXTRACT AS A NATURAL COLORING AGENT
}

H Anisa, Y Sukmawardani, and N Windayani (a)

\author{
Department of Chemistry Education, UIN Sunan Gunung Djati Bandung \\ JI. A. H. Nasution No. 105, Bandung 40614, Indonesia \\ a)E-mail: nenengwinda.ftk[at]uinsgd.ac.id
}

\begin{abstract}
This study aims to obtain an optimum combination formulation of cera alba in the manufacture of lip balm with natural material collorant in accordance with SNI. The experimental method used was maceration of natural products with $\mathrm{n}$-hexane solvents and concentrated with a rotary evaporator to find a dark yellow paste. Furthermore, making lip balm with various formulations of $2 \mathrm{gr}$ cera alba, $2.5 \mathrm{gr}, 3 \mathrm{gr}$ and without cera alba in $10 \mathrm{gr}$ shea butter with additives of orange and stawbery essence and natural coloring agent from carrots. The stability of lip blam the homogeneity test was carried out, the stability test carried out for 20 days at room temperature by observing changes in color, odor, and dosage form, $\mathrm{pH}$ test, topical test, and irritation test. The experimental results showed that all lip balm preparations were homogeneous, had a pH of 5-5,5 and did not cause skin irritation after 10 minutes of applying to the skin.
\end{abstract}

Keywords: Lip balm formulation, natural coloring agent, carrot extract 




\section{REFERENCES}

Abdillah, R., \& Setiawan, A. R. (2019). Topik Matematika dalam Taqrīb.

Abdillah, R., \& Setiawan, A. R. (2019). Topik Matematika yang Diperlukan Untuk Mengerti Taqrīb.

Agustina, D. (2017). PENERAPAN PEMBELAJARAN BERBASIS STEM (SCIENCE, TECHNOLOGY, ENGINEERING AND MATHEMATICS) UNTUK MENINGKATKAN SCIENTIFIC REASONING SISWA SMP PADA HUKUM PASCAL (Doctoral dissertation, Universitas Pendidikan Indonesia).

Agustina, Dessy, \& Setiawan, A. R. (2019, October 6). Building Students' Characters through STEM Education and Scientific Literacy. INA-Rxiv. DOI: https://doi.org/10.31227/osf.io/qtfnc

Fitriani, D. (2017). PEMBELAJARAN BERBASIS STEM (SCIENCE, TECHNOLOGY, ENGINEERING AND MATHEMATICS) PADA KONSEP TEKANAN HIDROSTATIS UNTUK MENINGKATKAN SCIENTIFIC REASONING SISWA SMP (Doctoral dissertation, Universitas Pendidikan Indonesia).

Jazil, S., \& Setiawan, A. R. (2019). Perbandingan Matan al-Ājrūmiyyah Ibn Ājurrūm dan Naḍhom al-Khulāșhoh al-Alfiyyah Ibn Mālik.

Khakim, M. L., \& Setiawan, A. R. (2019). Ḥisāb dan Ru’yat Waktu Ṣholāt Maktūbah di Daerah Tropis.

Koimah, S., \& Setiawan, A. R. (2019). A glance overview of the living environment.

Koimah, S., \& Setiawan, A. R. (2019). Effective Learning and Teaching.

Koimah, Siti, \& Setiawan, A. R. (2019, September 23). A Glance Overview of the Living Environment. Thesis Commons. DOI: https://dx.doi.org/10.31237/osf.io/6wyq4

Koimah, Siti; \& Setiawan, Adib Rifqi. (2019, September 23). A Glance Overview of the Living Environment. Thesis Commons. DOI: https://dx.doi.org/10.31237/osf.io/6wyq4

Kurniawati, N. K., \& Setiawan, A. R. (2019). Formulating the Purpose of Education Through Traditional Islamic Perspective and Literacy Competencies.

Louisa, G. N., \& Setiawan, A. R. (2019). Beda Kami-PSI-dengan Partai Lain.

Louisa, G. N., \& Setiawan, A. R. (2019). Keadilan Untuk Semua, Keadilan Untuk Perempuan Indonesia.

Louisa, G. N., \& Setiawan, A. R. (2019). Musuh Utama Persatuan Indonesia.

Louisa, G. N., \& Setiawan, A. R. (2019). Politik Akal Sehat, Politik Kaum Muda.

Louisa, G. N., \& Setiawan, A. R. (2019). Q \& A. Grace Natalie Louisa.[interview].

Maulana, I. R., \& Setiawan, A. R. (2019). Kondisi Alam Pada Awal Waktu Șholāt Maktūbah Di Daerah Tropis.

Maulana, M. S., \& Setiawan, A. R. (2019). Perbedaan Uraian Naḥwu dalam Matan al-Ājrūmiyyah Ibn Ājurrūm dan Naḍhom al-Khulāșhoh al-Alfiyyah Ibn Mālik.

Mughni, N. T. F., \& Setiawan, A. R. (2019). Perbandingan Corak Penafsiran antara Imām Jalāl alDīn al-Mahallī dan Imām Jalāl al-Dīn al-Suyūțī dalam Tafsīr al-Jalālayn.

Mughni, N. T. F., \& Setiawan, A. R. (2019). Perbandingan Penafsiran antara Imām Jalāl al-Dīn alMaḥallī dan Imām Jalāl al-Dīn al-Suyūțī dalam Tafsīr al-Jalālayn.

Mughni, N. T. F., \& Setiawan, A. R. (2019). The Comparison of Interpretation Characteristic Between Imām Jalāl Al-dīn Al-maḥalli Dan Imām Jalāl Al-dīn Al-suyūțī in Tafsīr Aljalālayn.

Musfiroh, M., \& Setiawan, A. R. (2019). The Value of the Persatuan Islam (PERSIS).

Nasriva, W. N., \& Setiawan, A. R. (2019). Analisis keabsahan dan keandalan science motivation questionnaire ii (smq ii) versi bahasa indonesia untuk pembelajaran bahasa arab.

Rianzi, A. F., \& Setiawan, A. R. (2019). Ideologi Patriarkal dalam iklan 'Segar Sari Susu Soda'versi Julia Perez.

Rianzi, A. F., Safaningrum, A. C., \& Setiawan, A. R. (2019). Konstruksi Bentuk Tubuh Perempuan dalam Iklan Julia Perez.

Safaningrum, A. C., \& Setiawan, A. R. (2019). Representasi Perempuan dalam iklan 'Kopi Susu Ya!'versi Julia Perez.

Safaningrum, A. C., Rianzi, A. F., Zein, L. F., \& Setiawan, A. R. (2019). How Julia Perez Use Her Erotic Capital?.

Setiawan, A. R. (2013). Bathari Ekonomi: tak langsir dirisak penyinyir. Alobatnic. blogspot. com.

Setiawan, A. R. (2013). Cendekiawan Picisan: melintang lintang intan terabaikan. Alobatnic. blogspot. com.

Setiawan, A. R. (2013). Kirana Tanpa Sirna: sepanjang menggelinjang memahat maslahat. Alobatnic. blogspot. com.

Setiawan, A. R. (2013). Menjilati Yuli: tegap menghibur walau dalam tangis. Alobatnic. blogspot. com.

Setiawan, A. R. (2013). Menjilati Yuli: tegap menghibur walau dalam tangis. 
Setiawan, A. R. (2013). Pangeran Ternistakan: kontroversi serta ambisi tiada henti. Alobatnic. blogspot. com.

Setiawan, A. R. (2014). Glam Male: kirana bunga manusia biasa. Alobatnic. blogspot. com.

Setiawan, A. R. (2014). Karena Lelaki Bukan Pengecut: driven by you tie your mother down. Alobatnic. blogspot. com.

Setiawan, A. R. (2014). Membaca Julia: karya biasa saja dari yuli rachmawati. Kirana $\mathscr{V}$ Azalea.

Setiawan, A. R. (2014). Popularitas adalah Tuhan: memberikan penghiburan, menumbuhkan pengharapan. Alobatnic. blogspot. com.

Setiawan, A. R. (2014). Risalah Kasidah: bayang bayang satu sisi restoe boemi. Alobatnic. blogspot. com.

Setiawan, A. R. (2015). Butcah Mbeling: the mad one's behavior in motion. Alobatnic. blogspot. com.

Setiawan, A. R. (2015). Melantan Warisan Kaisar Telantar: telantar, telantar, telantar, terlantan. Alobatnic. blogspot. com.

Setiawan, A. R. (2016). Dari Yuli Hingga Julia: memuji yuli, memuja julia. Kirana $\mathscr{V}$ Azalea.

Setiawan, A. R. (2016). Ionia: lingkungan pemula gelora kajian keilmuan. Alobatnic. blogspot. com.

Setiawan, A. R. (2016). K2: mengenang sahabat yang hebat. Alobatnic. blogspot. com.

Setiawan, A. R. (2016). Kamu dalam aku. Kirana $\mathscr{P}$ Azalea.

Setiawan, A. R. (2016). Kamu Dalam aku: memuja yang dicinta, membela yang dinista. Alobatnic. blogspot. com.

Setiawan, A. R. (2016). Mike Shinoda. Alobatnic. blogspot. com.

Setiawan, A. R. (2016). PARIS: nothing in this world turn you on. Alobatnic. blogspot. com.

Setiawan, A. R. (2017). Butcah Chuniez.

Setiawan, A. R. (2017). Butcah Chuniez: Puzzle Persinggungan Perjalanan dengan Eny Rochmwati Octaviani. Alobatnic. blogspot. com.

Setiawan, A. R. (2017). Guru yang Menyapih: tak melayang dipuji, tak tumbang dicaci. Alobatnic. blogspot. com.

Setiawan, A. R. (2017). Hope, Faith, Love: A Brief Story of Yuli Rahmawati. Alobatnic. blogspot. com.

Setiawan, A. R. (2017). Hope, Faith, Love: A Brief Story of Yuli Rahmawati.

Setiawan, A. R. (2017). Itz Spring Voice: andai aku tak mengaguminya. Alobatnic. blogspot. com.

Setiawan, A. R. (2017). Meniti Ilmuwati: catatan peran perempuan dalam pembahasan alam. Alobatnic. blogspot. com.

Setiawan, A. R. (2017). My Way Rhapsody!: Islam Aries-Blackjack, Is Islam?. Alobatnic. blogspot. com.

Setiawan, A. R. (2017). My Way Rhapsody. Islam Aries-Blackjack, Is Islam.

Setiawan, A. R. (2018). A Brief Note of Science Education Development in Indonesia. Alobatnic. blogspot. com.

Setiawan, A. R. (2018). Aisha: rebel heart living for love. Alobatnic. blogspot. com.

Setiawan, A. R. (2018). Amazing Grace: Satu Sisi Religi Grace Natalie. Alobatnic. blogspot. com.

Setiawan, A. R. (2018). Apresiasi Semadyana pada YoonA: Ketika YoonA mampir ke Parc des Princes. Alobatnic. blogspot. com.

Setiawan, A. R. (2018). Bergerak Menuju Tak Tentu: risalah rị̣lah khidmah'ilmiyyah. Alobatnic. blogspot. com.

Setiawan, A. R. (2018). Breast Capital: Konsep Baru Duo Serigala, Modal Erotis, dan Payudara Perempuan. Alobatnic. blogspot. com.

Setiawan, A. R. (2018). Breast Capital: Konsep Baru Duo Serigala, Modal Erotis, dan Payudara Perempuan. Alobatnic. blogspot. com. URL: https:// Alobatnic. blogspot. com/2018/03/breastcapital. html.

Setiawan, A. R. (2018). Busana: pemantas raga, pelaras jiwa. Alobatnic. blogspot. com.

Setiawan, A. R. (2018). Clara Ng: an author for our time. Alobatnic. blogspot. com.

Setiawan, A. R. (2018). Come Back Bom![컴백봄!]. Alobatnic. blogspot. com.

Setiawan, A. R. (2018). Crazy Rhapsody: deep in my heartCrazy Rhapsody: deep in my heart. Alobatnic. blogspot. com.

Setiawan, A. R. (2018). Dari Fallen Menjadi Vallen: Fall in Love Vallen in Love in Valentine. Alobatnic. blogspot. com.

Setiawan, A. R. (2018). Debut Mengajar Biologi: mengenali diri, melantan lingkungan. Alobatnic. blogspot. com.

Setiawan, A. R. (2018). Drama korea. Alobatnic. blogspot. com.

Setiawan, A. R. (2018). Fakhrun: a brief friendship (5 November 11-2 Agustus 2014). Alobatnic. blogspot. com.

Setiawan, A. R. (2018). Girls, Love, War: A Brief Story of Rahwayana. Alobatnic. blogspot. com. 
Setiawan, A. R. (2018). Grace Natalie, PSI, Poligami. Alobatnic. blogspot. com.

Setiawan, A. R. (2018). Guru yang Menyapih: Catatan Perjalanan Pribadi Nong Darol Mahmada.

Setiawan, A. R. (2018). Hard to say happy birthday park bom. Alobatnic. blogspot. com.

Setiawan, A. R. (2018). Hukum Alam, Kehendak Bebas, Perilaku Manusia. Alobatnic. blogspot. com.

Setiawan, A. R. (2018). Into the New World (다시 만난 세계): proposal for the principle of least action in the simple life. Alobatnic. blogspot. com.

Setiawan, A. R. (2018). Irma Rahma Suwarma. Alobatnic. blogspot. com.

Setiawan, A. R. (2018). Islām Aries-Blackjack, is Islām?. Alobatnic. blogspot. com.

Setiawan, A. R. (2018). Julia Perez-Iklan Segar Sari Susu Soda. YouTube. com/AdibRS.

Setiawan, A. R. (2018). Julia Perez-Iklan Ya Kopi Susu. YouTube. com/AdibRS.

Setiawan, A. R. (2018). Karen Armstrong: there is a rainbow in Islām. Alobatnic. blogspot. com.

Setiawan, A. R. (2018). Ketika Ibrōhīm Mengajak untuk Mengamati Alam: dari dorongan penasaran sampai gairah berserah. Alobatnic. blogspot. com.

Setiawan, A. R. (2018). Ki Oza Kioza.

Setiawan, A. R. (2018). Ki Oza Kioza: A Rain Shine Made in Indonesia. Alobatnic. blogspot. com.

Setiawan, A. R. (2018). Kisah Hidup Lola Zieta. Soundcloud. com/femocrazy.

Setiawan, A. R. (2018). Laila Fariha Zein (aka Uus atau Febi): penyuluh peluh, peluluh keluh, peluruh keruh. Alobatnic. blogspot. com.

Setiawan, A. R. (2018). Lola Zieta Azelien: paduan yakin diri dan rendah hati memanfaatkan kecantikan. Alobatnic. blogspot. com.

Setiawan, A. R. (2018). Mabādī 'Asyroh isika. Alobatnic. blogspot. com.

Setiawan, A. R. (2018). Madrasah Tasywiquth Thullab Salafiyyah (TBS) Kudus. Alobatnic. blogspot. com.

Setiawan, A. R. (2018). Manunggaling Meyda Sefira-Hujan Safir. Alobatnic. blogspot. com.

Setiawan, A. R. (2018). Mathematics: to write nor not to write. Alobatnic. blogspot. com.

Setiawan, A. R. (2018). Máthēmatnic: catatan perjalanan. Alobatnic. blogspot. com.

Setiawan, A. R. (2018). Menelusuri Nama Maryam: anyam sanggam, anyam azam. Alobatnic. blogspot. com.

Setiawan, A. R. (2018). Modal erotis payudara perempuan. Qureta. com.

Setiawan, A. R. (2018). Pain Man or Fine Man. Apa sih yang Istimewa dari Richard Phillips Feynman.

Setiawan, A. R. (2018). Pain Man or Fine Man?: Apa sih yang Istimewa dari Richard Phillips Feynman. Alobatnic. blogspot. com.

Setiawan, A. R. (2018). Pantat Perekat Umat: Modal Erotic Zaskia Gotik. Alobatnic. blogspot. com.

Setiawan, A. R. (2018). Paul DeHart Hurd: menjadi-jadi dari biologi. Alobatnic. blogspot. com.

Setiawan, A. R. (2018). Penerapan Pendekatan Saintifik untuk Melatih Literasi Saintifik dalam Domain Kompetensi pada Topik Gerak Lurus di Sekolah Menengah Pertama. In Seminar Nasional Fisika (Vol. 4, No. 1, pp. 7-13).

Setiawan, A. R. (2018). Peran Biologi dalam Mendukung Pelaksanaan Jihād. Alobatnic. blogspot. com.

Setiawan, A. R. (2018). Remy Sylado 23761: konsisten dengan seribu kemuliaan. Alobatnic. blogspot. com.

Setiawan, A. R. (2018). Risalah Rị̣lah (Catatan Penjelajahan): melestarikan tradisi bermadzhab. Alobatnic. blogspot. com.

Setiawan, A. R. (2018). Rosa Amalia Iqony: paduan yakin diri dan rendah hati. Alobatnic. blogspot. com.

Setiawan, A. R. (2018). Segitiga Sama Cinta: who is the queen of the sciences: mathematics or biology?. Alobatnic. blogspot. com.

Setiawan, A. R. (2018). Sekilas Mengelilingi Luas Geometri: Mengingat René Descartes, Mengenang Muhammad al-Ghazālī. Alobatnic. blogspot. com.

Setiawan, A. R. (2018). Six Not-So-Amazing Video. Alobatnic. blogspot. com.

Setiawan, A. R. (2018). Surely You're Joking, Mr Feynman.

Setiawan, A. R. (2018). Surely You're Joking, Mr. Feynman!: Adventures of a Curious Character. Alobatnic. blogspot. com.

Setiawan, A. R. (2018). This is Venice: tak lelah meniti tatanan, tak jengah menata titian. Alobatnic. blogspot. com.

Setiawan, A. R. (2018). Venice Min: yakin diri dan rendah hati memanfaatkan kecantikan. Alobatnic. blogspot. com.

Setiawan, A. R. (2019). A Biography of Eny Rochmawati Octaviani.

Setiawan, A. R. (2019). A Brief Commentary on Kirsten Stadermann of Groningen's Paper.

Setiawan, A. R. (2019). A Brief Explanation of Scientific Teaching.

Setiawan, A. R. (2019). A Brief Overview of Singapore Mathematics Syllabus. 
Setiawan, A. R. (2019). A Briefest Historical Trajectory of Kitab Kuning.

Setiawan, A. R. (2019). A Glance History of Physical Science.

Setiawan, A. R. (2019). A Glance of Elementary Mathematics's Portrayal.

Setiawan, A. R. (2019). A Glance Overview of Ecofeminism.

Setiawan, A. R. (2019). A Glance Overview of Light Pollution.

Setiawan, A. R. (2019). A Glance Portrayal of Science Education in Indonesia.

Setiawan, A. R. (2019). A Glance PrePrints's Portrayal.

Setiawan, A. R. (2019). A Glance PrePrints's Portrayal: based on two months damn experiences of dumb user.

Setiawan, A. R. (2019). A Leader for Our Time: Ulasan Biografi Grace Natalie Louisa.

Setiawan, A. R. (2019). Aligning Education for Sustainable Development (ESD) and Traditional Islamic Perspective.

Setiawan, A. R. (2019). Amazing Grace: Sisi Religi Grace Natalie Louisa Ketua Umum Partai Solidaritas Indonesia.

Setiawan, A. R. (2019). An Interview with Amber Chia.

Setiawan, A. R. (2019). An Interview with the Physicist that Her Head in the Universe and Both Feet on the Earth.

Setiawan, A. R. (2019). Apa yang dapat dilakukan dalam pembelajaran Taqrīb?.

Setiawan, A. R. (2019). Assessment for Ecological Learning with Scientific Literacy Oriented.

Setiawan, A. R. (2019). Bagaimana Sekolah Menengah Mengajar Fisika Kuantum?.

Setiawan, A. R. (2019). Bercerita cerita clara.

Setiawan, A. R. (2019). Bergerak Menuju Tak Tentu.

Setiawan, A. R. (2019). Biografi Eny Rochmawati Octaviani.

Setiawan, A. R. (2019). Blue Spring: A Brief Journey of Park Bom.

Setiawan, A. R. (2019). Breast Capital: A Glance Journey of Yuli Rahmawati as an Entertainer for Our Time.

Setiawan, A. R. (2019). Breast Capital: The Benefits of Erotic Capital Through the Career of Duo Serigala.

Setiawan, A. R. (2019). Cathryn Li Yuan Ling: déesse séduit Internet avec sa multi-maîtrise.

Setiawan, A. R. (2019). Clara Ng: A Brief Journey of A Remarkable Female for Our Time.

Setiawan, A. R. (2019). Clara Ng: Un Bref Voyage d'Une Femme Remarquable pour Notre Temps.

Setiawan, A. R. (2019). Comparing Normalized Gain g and Effect Size Cohen'sd to Measure the Improvement of Student's Scientific Literacy.

Setiawan, A. R. (2019). Constructing Assessment Instrument for Scientific Literacy's Oriented Learning in Elementary Education.

Setiawan, A. R. (2019). Constructing Thematic Learning Lesson Plan to Guide Primary Education Student on Achieving Scientific Literacy.

Setiawan, A. R. (2019). Constructing Thematic Learning Lesson Plan for Guide Students on Achieving Scientific Literacy.

Setiawan, A. R. (2019). Contoh rancangan pembelajaran fisika berorientasi hots (higher order thinking skill).

Setiawan, A. R. (2019). Curahan Hati untuk Teman-Teman Santri.

Setiawan, A. R. (2019). Dari Kurikulum Ke Kurikulum.

Setiawan, A. R. (2019). Designing A Software for Processing Pencil-Based Instrument of Learning Assessment.

Setiawan, A. R. (2019). Designing an islamic studies curriculum: aligning the purpose of education and learning activities through traditional islamic perspective and scientific literacy competencies.

Setiawan, A. R. (2019). Designing an Islamic Studies Curriculum: Aligning the Purpose of Education, Learning Contents, and Syllabus Through Traditional Islamic Perspective and Literacy Competencies.

Setiawan, A. R. (2019). Developing STEM Education's Lesson Plan for Guide Students to Achieve Scientific Literacy.

Setiawan, A. R. (2019). Education for Sustainable Development (ESD).

Setiawan, A. R. (2019). Efektivitas pembelajaran biologi berorientasi literasi saintifik.

Setiawan, A. R. (2019). Effectiveness of biology learning scientific literacy oriented.

Setiawan, A. R. (2019). Ekspresi Perempuan dalam 'Kill This Love'dari BLACKPINK.

Setiawan, A. R. (2019). Employing Maba'di' 'Asyroh to Elaborate Nature of Science.

Setiawan, A. R. (2019). Eny R. Octaviani: A Role Model for Our Time.

Setiawan, A. R. (2019). Eny Rochmawati Octaviani.

Setiawan, A. R. (2019). Female Beauty in "As If It's Your Last" from BLACKPINK.

Setiawan, A. R. (2019). Fenny Roshayanti: A Biography of Science Educator for Our Time.

Setiawan, A. R. (2019). Grace Natalie Louisa. 
Setiawan, A. R. (2019). Grace Natalie, PSI, Poligami: Sisi Feminis Grace Natalie Louisa Ketua Umum Partai Solidaritas Indonesia.

Setiawan, A. R. (2019). Guru yang Menyapih: Biografi Pribadi Nong Darol Mahmada.

Setiawan, A. R. (2019). Her Majesty Queen Rania Al Abdullah.

Setiawan, A. R. (2019). How Girls Generation Use Erotic Capital?.

Setiawan, A. R. (2019). How to Choose Our Study?.

Setiawan, A. R. (2019). How to Teach and Do Research in Physics Education. Open Science Framework (OSF) Preprints.

Setiawan, A. R. (2019). Hubungan Fisika dan Matematika dalam Pembelajaran Aktual.

Setiawan, A. R. (2019). Implementation of Scientific Teaching to Train Scientific Literacy in Domain Competence at Lower Secondary School in Topic Linear Motion.

Setiawan, A. R. (2019). Instrumen penilaian untuk pembelajaran ekologi berorientasi literasi saintifik.

Setiawan, A. R. (2019). Integrasi Kaidah Fiqih dalam Pembelajaran Ekologi.

Setiawan, A. R. (2019). Irma Rahma Suwarma: A Science Educator for Our Time. LIS Scholarship Archive (LISSA).

Setiawan, A. R. (2019). Is this Educational World We Created?.

Setiawan, A. R. (2019). Jessica Jung: three decades of the modern-classic aesthetic sequencing. Setiawan, A. R. (2019). Kecantikan Perempuan dalam "As If It's Your Last" dari BLACKPINK.

Setiawan, A. R. (2019). Laila Fariha Zein.

Setiawan, A. R. (2019). Laila Fariha Zein: A Teachress for Our Time.

Setiawan, A. R. (2019). LOLA ZIETA AZELIEN: a boy with a pair of boobs.

Setiawan, A. R. (2019). Maryam Musfiroh: An Educator for Our Time.

Setiawan, A. R. (2019). Máthēmatnic: pengalaman memandu pembelajaran matematika.

Setiawan, A. R. (2019). Membaca 'te-ko Ramadan 1440'Karya Laila Fariha Zein.

Setiawan, A. R. (2019). Mengikuti Kolokium Atau Menerbitkan Jurnal?.

Setiawan, A. R. (2019). Meniti Ilmuwati. Open Science Framework.

Setiawan, A. R. (2019). Menyusun Soal Literasi Saintifik Topik Gerak Lurus untuk Sekolah Menengah.

Setiawan, A. R. (2019). Menyusun Tes Pembelajaran Tematik untuk Pendidikan Dasar.

Setiawan, A. R. (2019). M^M^.

Setiawan, A. R. (2019). Nong Darol Mahmada: Guru yang Menyapih.

Setiawan, A. R. (2019). Observing the Interethnic Friendships in Elementary and Secondary Religion-Based School.

Setiawan, A. R. (2019). Oza Kioza.

Setiawan, A. R. (2019). Oza Kioza: dari pita suara sampai payudara.

Setiawan, A. R. (2019). Pandangan Nong Darol Mahmada terhadap Pemakaian Jilbab.

Setiawan, A. R. (2019). Panduan Penulisan Artikel Akademik.

Setiawan, A. R. (2019). Pantat Perekat Umat.

Setiawan, A. R. (2019). Pantat Perekat Umat: Kekuatan Modal Erotis Sepanjang Karier Zaskia Gotik.

Setiawan, A. R. (2019). Paris, Not Française: A Brief Journey of The Simple Person.

Setiawan, A. R. (2019). Pendidikan untuk Pembangunan Berkelanjutan.

Setiawan, A. R. (2019). Pengalaman Memandu Pembelajaran Matematika di MPTs NU TBS Kudus.

Setiawan, A. R. (2019). Penggunaan Naḍom Mabađili'Asyroh dalam Pembelajaran Biologi untuk Meningkatkan Motivasi dan Hasil Belajar.

Setiawan, A. R. (2019). Penyusunan Program Pembelajaran Biologi Berorientasi Literasi Saintifik. Setiawan, A. R. (2019). Per (empu) an.

Setiawan, A. R. (2019). Peran Tanaman dalam Mengurangi Polusi Lingkungan di Kompleks Perguruan Tinggi Kecamatan Lowokwaru Kota Malang.

Setiawan, A. R. (2019). Perihal Kehendak Manusia.

Setiawan, A. R. (2019). Personal Interview with Venice Min.

Setiawan, A. R. (2019). Playing With Fire-essentials mathematics explained by dumb practical man based on damn experience.

Setiawan, A. R. (2019). Princess Lexie: A Glance Journey of A Princess for Our Time.

Setiawan, A. R. (2019). Puan Maharani: A Women's Representative in the Politics of Indonesia.

Setiawan, A. R. (2019). Q \& A. Lisa Randall.[interview].

Setiawan, A. R. (2019). Risalah Ilmiyah: Adventures with a Curious Leader.

Setiawan, A. R. (2019). Rosa Amalia Iqony: How Muslimah Use Her Erotic Capital?.

Setiawan, A. R. (2019). ROSÉ.

Setiawan, A. R. (2019). Science Motivation Questionnaire II (smq-ii): analysis of Validity and Reliability of Bahasa Indonesia Version in Science Learning Context. 
Setiawan, A. R. (2019). Scientific Literacy Profile Based on Multiple Intelligences and Learning Motivation Through Thematic Learning in Primary Education.

Setiawan, A. R. (2019). Sekilas tentang Pendidikan Sains di Indonesia.

Setiawan, A. R. (2019). Sekilas tentang Polusi Cahaya.

Setiawan, A. R. (2019). Sex, Love, Faith: A Brief Story of Yuli Rahmawati aka Jupe.

Setiawan, A. R. (2019). Silabus Pembelajaran Karya Tulis Pondok Pesantren Ath-Thullab.

Setiawan, A. R. (2019). Sisi Feminis Perempuan Korea Selatan dalam album 'To Anyone'dari 2 NE1.

Setiawan, A. R. (2019). Six raisons simples: Principes de base de l'enseignement des sciences (naturelles) en Indonésie.

Setiawan, A. R. (2019). Six Rhetoric Quadratic: the Six Types of Quadratic Equations Presented by Abū Ja'far Muhammad ibn Mūsā al-Khwārizmī in al-Kitāb al-Mukhtașor fĩ Hisāb alJabr wa al-Muqōbala.

Setiawan, A. R. (2019). Six Simple Reasons.

Setiawan, A. R. (2019). Soal HOTS Akidah Akhlak untuk Kelas 4 Madrasah Ibtidaiyah.

Setiawan, A. R. (2019). STEM Education Problem Through Striking Differences Between Physics and Biology.

Setiawan, A. R. (2019). Sunmi.

Setiawan, A. R. (2019). Tak Melayang Dipuji, Tak Tumbang Dicaci: Kajian Biografi Oza Kioza.

Setiawan, A. R. (2019). The Conservative Side of the Natural Science.

Setiawan, A. R. (2019). The Difference Between Normalized Gain g and Effect Size Cohen'sd for Measuring the Improvement of Student's Scientific Literacy.

Setiawan, A. R. (2019). The Parasitism of Mathematics in Physics Learning.

Setiawan, A. R. (2019). The Quotable Laila Fariha Zein.

Setiawan, A. R. (2019). The Relation of Science and al-Qur'ān: a Personal Perspective on an Old Topic.

Setiawan, A. R. (2019). Thematic Learning Brief Explanation.

Setiawan, A. R. (2019). Upaya Melatih Literasi Saintifik melalui Pembelajaran Ilmu Pengetahuan Alam.

Setiawan, A. R. (2019). Upaya meningatkan motivasi belajar matematika menggunakan topik fiqih dan astronomi.

Setiawan, A. R. (2019). Upaya Meningkatkan Motivasi Belajar dalam Pembelajaran Ilmu Pengetahuan Alam (IPA) Melalui Bacaan Populer.

Setiawan, A. R. (2019). Venice min: a model for our time.

Setiawan, A. R. (2019). Villa Isola.

Setiawan, A. R. (2019). Whasfi Velasufah: A Biography of a Female Activists.

Setiawan, A. R. (2019). رسَالَةُ الْعَلْيَيَّة .

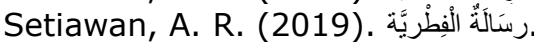

Setiawan, A. R. (2019). مَبَادِى علم الأحياء للمدرسة العالية تشويق الطلاب سِلفِّيَّة .

Setiawan, A. R. (2019, July 08). A Glance Overview of Light Pollution. Open Science Framework. DOI: https://dx.doi.org/10.31219/osf.io/m8ay2

Setiawan, A. R. (2019, Juy 07). Sekilas tentang Polusi Cahaya. INA-Rxiv. DOI: https://dx.doi.org/10.31227/osf.io/9ft5x

Setiawan, A. R. (2019, October 04). Eny Rochmawati Octaviani. Thesis Commons. DOI: https://dx.doi.org/10.31237/osf.io/g5zf6

Setiawan, A. R. (2019, October 04). The Difference Between Hake's Normalized Gain g and Effect Size Cohen's d for Measuring the Improvement of Student's Scientific Literacy. EdArXiv. DOI: https://dx.doi.org/10.35542/osf.io/fxu4t

Setiawan, A. R. (2019, October 05). Menyusun Instrumen Penilaian untuk Pembelajaran Ekologi Berorientasi Literasi Saintifik. EdArXiv. DOI: https://dx.doi.org/10.35542/osf.io/rhzqa

Setiawan, A. R. (2019, October 2). Breast Capital: A Glance Journey of Yuli Rahmawati as an Entertainer for Our Time. Thesis Commons. DOI: https://dx.doi.org/10.31237/osf.io/wp87n

Setiawan, A. R. (2019, October 2). Puan Maharani: A Women's Representative in the Politics of Indonesia. Thesis Commons. DOI: https://dx.doi.org/10.31237/osf.io/8b4xh

Setiawan, A. R. (2019, October 2). The Difference Between Normalized Gain g and Effect Size Cohen's d for Measuring the Improvement of Student's Scientific Literacy. Thesis Commons. DOI: https://dx.doi.org/10.31237/osf.io/vrwbj

Setiawan, A. R. (2019, October 4). The Difference Between Hake's Normalized Gain g and Effect Size Cohen's d for Measuring the Improvement of Student's Scientific Literacy. EdArXiv. DOI: https://doi.org/10.35542/osf.io/fxu4t

Setiawan, A. R. (2019, October 8). Biografi Clara Ng Perempuan Penulis Asal Indonesia. PsyArXiv. DOI: https://doi.org/10.31234/osf.io/yk8sx 
Setiawan, A. R. (2019, October 8). Kurikulum Lokal Madrasah Tasywiquth Thullab Salafiyyah (TBS) Kudus. Thesis Commons. DOI: https://doi.org/10.31237/osf.io/2m6pd

Setiawan, A. R. (2019, October 8). Penerapan Pendekatan Saintifik dalam Pembelajaran Biologi Berorientasi Literasi Saintifik. EdArXiv. DOI: https://doi.org/10.35542/osf.io/4nas8

Setiawan, A. R. (2019, October 8). Penerapan Pendekatan Saintifik dalam Pembelajaran Biologi Berorientasi Literasi Saintifik. Thesis Commons. DOI: https://doi.org/10.31237/osf.io/dua9t

Setiawan, A. R. (2019, October 8). The Implementation of Scientific Approach in Biology Learning to Guide Students on Achieve Scientific Literacy. Thesis Commons. DOI: https://doi.org/10.31237/osf.io/rceq6

Setiawan, A. R. (2019, October 8). The Implementation of Thematic Learning to Guide Students on Achieve Scientific Literacy. Thesis Commons. DOI: https://doi.org/10.31237/osf.io/zmf6p

Setiawan, A. R. (2019, October). Menyusun Instrumen Penilaian untuk Pembelajaran Ekologi Berorientasi Literasi Saintifik. In Seminar Nasional dan Workshop Biologi IPA dan Pembelajarannya ke-4 dengan tema "BioGlocal" di Jurusan Biologi Fakultas Matematika, IImu Pengetahuan Alam, dan Teknologi Informasi Universitas Negeri Malang pada (Vol. 5).

Setiawan, A. R. (2019, September 17). Fenny Roshayanti: A Biography of Science Educator for Our Time. Thesis Commons. DOI: https://dx.doi.org/10.31237/osf.io/37une

Setiawan, A. R. (2019, September 17). Six Rhetoric Quadratic: the Six Types of Quadratic Equations Presented by Abū Ja'far Muḥammad ibn Mūsā al-Khwārizmī in al-Kitāb al-Mukhtașor fị Hiisāb al-Jabr wa al-Muqōbala. Thesis Commons. DOI: https://dx.doi.org/10.31237/osf.io/pn3dx

Setiawan, A. R. (2019, September 20). Designing an Islamic Studies Curriculum: Aligning the Purpose of Education and Learning Activities Through Traditional Islamic Perspective and Scientific Literacy Competencies. Thesis Commons. DOI: https://dx.doi.org/10.31237/osf.io/5d3tq

Setiawan, A. R. (2019, September 20). The Relation of Science and al-Qur'ān: a Personal Perspective on an Old Topic. Thesis Commons. DOI: https://dx.doi.org/10.31237/osf.io/yu9mz

Setiawan, A. R. (2019, September 21). Whasfi Velasufah: A Biography of a Female Activists. Thesis Commons. DOI: https://dx.doi.org/10.31237/osf.io/fyrjd

Setiawan, A. R. (2019, September 22). Her Majesty Queen Rania Al Abdullah. Thesis Commons. DOI: https://dx.doi.org/10.31237/osf.io/thp42

Setiawan, A. R. (2019, September 23). Irma Rahma Suwarma: A Science Educator for Our Time. Thesis Commons. DOI: https://dx.doi.org/10.31237/osf.io/g9jst

Setiawan, A. R. (2019, September 24). Designing A Software for Processing Pencil-Based Instrument of Learning Assessment. Thesis Commons. DOI: https://dx.doi.org/10.31237/osf.io/tcq2a

Setiawan, A. R. (2019, September 25). Female Beauty in "As If It's Your Last" from BLACKPINK. Thesis Commons. DOI: https://dx.doi.org/10.31237/osf.io/m26cx

Setiawan, A. R. (2019, September 25). Kecantikan Perempuan dalam "As If It's Your Last" dari BLACKPINK. Thesis Commons. DOI: https://dx.doi.org/10.31237/osf.io/fh32w

Setiawan, A. R. (2019, September 26). Effectiveness of biology learning scientific literacy oriented. EdArXiv. DOI: https://dx,doi.org/10.35542/osf.io/yw74s

Setiawan, A. R. (2019, September 26). Observing the Interethnic Friendships in Elementary and Secondary Religion-Based School. Thesis Commons. DOI: https://dx.doi.org/10.31237/osf.io/mzvqu

Setiawan, A. R. (2019, September 26). The Effectiveness of Biology Learning Scientific Literacy Oriented. EdArXiv. DOI: https://dx.doi.org/10.35542/osf.io/8pgy9

Setiawan, A. R. (2019, September 28). Aligning Education for Sustainable Development (ESD) and Traditional Islamic Perspective. EdArXiv. DOI: https://dx.doi.org/10.35542/osf.io/gtsef

Setiawan, A. R. (2019, September 29). An Interview with the Physicist that Her Head in the Universe and Both Feet on the Earth. LIS Scholarship Archive. DOI: https://dx.doi.org/10.31229/osf.io/jdw7f

Setiawan, A. R. (2019, September 29). Constructing Thematic Learning Lesson Plan to Guide Primary Education Student on Achieving Scientific Literacy. EdArXiv. DOI: https://dx.doi.org/10.35542/osf.io/dn87g 
Setiawan, A. R. (2019, September 29). Scientific Literacy Profile Based on Multiple Intelligences and Learning Motivation Through Thematic Learning in Primary Education. Thesis Commons. DOI: https://dx.doi.org/10.31237/osf.io/xnjk8

Setiawan, A. R. (2019, September 30). An Interview with the Physicist that Her Head in the Universe and Both Feet on the Earth. INA-Rxiv. DOI: https://dx.doi.org/10.31227/osf.io/mfv4e

Setiawan, A. R. (2019, September 30). Assessment for Ecological Learning with Scientific Literacy Oriented. EdArXiv. DOI: https://dx.doi.org/10.35542/osf.io/kw4bq

Setiawan, A. R. (2019, September 30). Constructing Thematic Learning Lesson Plan to Guide Primary Education Student on Achieving Scientific Literacy. Thesis Commons. DOI: https://dx.doi.org/10.31237/osf.io/z4m6h

Setiawan, A. R., \& IImiyah, S. (2019). My Journey as A Part of CSS Mora UIN Syahid Jakarta. Setiawan, A. R., \& Inayati, F. (2019). A Glance Portrayal of Madrasahs in Singapore.

Setiawan, A. R., \& Inayati, F. (2019). Islam Nusantara: Glance History, Characteristics, and Criticism.

Setiawan, A. R., \& Inayati, F. (2019). Islam Nusantara: Glance History, Characteristics, and Criticism.

Setiawan, A. R., \& Koimah, S. (2019). Effective learning and teaching.

Setiawan, A. R., \& Koimah, Siti. (2019, September 23). Effective Learning and Teaching. Thesis Commons. DOI: https://dx.doi.org/10.31237/osf.io/p42nx

Setiawan, A. R., \& Louisa, G. N. (2018). Keadilan Untuk Semua, Keadilan Untuk Perempuan Indonesia. Alobatnic. blogspot. com.

Setiawan, A. R., \& Ma'munah, M. (2015). Nun: bahadur menghibur melantan pengharapan. Alobatnic. blogspot. com.

Setiawan, A. R., \& Min, V. (2019). An Interview with Venice Min.

Setiawan, A. R., \& Mughni, N. T. F. (2019). Hubungan Fisika dan Matematika dalam Kurikulum Indonesia.

Setiawan, A. R., \& Mughni, N. T. F. (2019). The Relation of Physics and Mathematics in Indonesia Curriculum.

Setiawan, A. R., \& Musfiroh, M. (2019). Pendidikan Karakter: Akhlak, Adab, Moral dan Nilai.

Setiawan, A. R., \& Octaviani, E. R. (2019). Yoona: A Brief Journey of An Entertainer for Our Time.

Setiawan, A. R., \& Sari, D. R. (2019). A Simple Essay of Natural Science Curricula in Indonesia.

Setiawan, A. R., \& Si'ayah, S. (2019). Mengurangi Polusi Suara Menggunakan Tanaman.

Setiawan, A. R., \& Si'ayah, S. (2019). Penggunaan Tanaman untuk Mengurangi Polusi Suara.

Setiawan, A. R., \& Si'ayah, S. (2019). Analisis Keabsahan Dan Keandalan Multiple Intelligences Survey (MIS) Versi Bahasa Indonesia.

Setiawan, A. R., \& Van Bee, J. L. (2019). Ethnomathematics at a Glance.

Setiawan, A. R., \& Velasufah, W. (2019). Islamic Perspective Toward Education for Sustainable Development (ESD).

Setiawan, A. R., \& Velasufah, Whasfi. (2019, September 28). Islamic Perspective Toward Education for Sustainable Development (ESD). Thesis Commons. DOI: https://dx.doi.org/10.31237/osf.io/qna2g

Setiawan, A. R., \& Yulidarti, S. (2019). Designing Worksheet to Guide Primary Education Student on Achieving Scientific Literacy.

Setiawan, A. R., \& Yulidarti, Sherly. (2019, September 29). Designing Worksheet to Guide Primary Education Student on Achieving Scientific Literacy. Thesis Commons. DOI: https://dx.doi.org/10.31237/osf.io/e27wq

Setiawan, A. R., \& Zein, L. F. (2019). The Value of René Descartes's Work Devoted to Princess Elisabeth of Bohemia in Psychology.

Setiawan, A. R., \& Zein, L. F. (2019). Un Essai Simple de Programmes de Sciences Naturelles en Indonésie.

Setiawan, A. R., Agustina, D., \& Fitriani, D. (2019). Designing STEM Education's Lesson Plan for Guide Students to Achieve Scientific Literacy.

Setiawan, A. R., Sari, D. R., Musfiroh, M., \& Iqony, R. A. (2019). A Short Description of Pondok Pesantren.

Setiawan, A. R., Zein, L. F., \& Safaningrum, A. C. (2019). Is 'god Is a Woman'from Ariana Grande Only a Song?.

Setiawan, Adib Rifqi. (2017, February 24). Penerapan Pendekatan Saintifik untuk Melatihkan Literasi Saintifik dalam Domain Kompetensi pada Topik Gerak Lurus di Sekolah Menengah Pertama. Undergraduate Thesis. Universitas Pendidikan Indonesia. URL: http://repository.upi.edu/29074/

Setiawan, Adib Rifqi. (2018, 24 November). Penerapan Pendekatan Saintifik untuk Melatih Literasi Saintifik dalam Domain Kompetensi pada Topik Gerak Lurus di Sekolah Menengah 
Pertama. Disampaikan dalam Seminar Nasional Fisika (SiNaFi) 2018 dengan tema "Membangun. Kemandirian dan Daya Saing Bangsa melalui Pendidikan dan Penelitian Fisika" di Departemen Pendidikan Fisika Universitas Pendidikan Indonesia (UPI) Bandung pada 24 November 2018. DOI: https://dx.doi.org/10.13140/RG.2.2.23154.04809

Setiawan, Adib Rifqi. (2018, April 10). Busana: pemantas raga, pelaras jiwa. Majalah SANTRI, 8: 26-27. URL: https://issuu.com/majalahsantri/docs/majalah_santri_8/28

Setiawan, Adib Rifqi. (2018, April 10). Eny Rochmawati Octaviani: memberikan hiburan, menyuntikkan harapan. Majalah SANTRI, 8: 15-18.

URL: https://issuu.com/majalahsantri/docs/majalah_santri_8/17

Setiawan, Adib Rifqi. (2018, April 10). Rosa Amalia Iqony: paduan yakin diri dan rendah hati. Majalah SANTRI, 8: 46.

URL: https://issuu.com/majalahsantri/docs/majalah_santri_8/48

Setiawan, Adib Rifqi. (2019, 05 Oktober). Menyusun Instrumen Penilaian untuk Pembelajaran Ekologi Berorientasi Literasi Saintifik. Disampaikan dalam Seminar Nasional \& Workshop Biologi, IPA, dan Pembelajarannya dengan tema "Transformasi Pendidikan/Pembelajaran Sains-Biologi Abad 21 untuk mendukung Education for Sustainable Development" di Jurusan Biologi Universitas Negeri Malang pada 05 Oktober 2019. DOI: https://dx.doi.org/10.13140/RG.2.2.21931.39206

Setiawan, Adib Rifqi. (2019, August 21). Penyusunan Program Pembelajaran Biologi Berorientasi Literasi Saintifik. Disampaikan dalam Seminar Nasional Sains \& Entrepreneurship IV (SNSE IV) dengan tema "Transformasi Sains dalam Pembelajaran untuk Menyiapkan SDM Pengelola Sumber Daya Hayati Berkelanjutan di Era Revolusi Industri 4.0 Bervisi Entrepreneurship" di Program Studi Pendidikan Biologi Universitas PGRI Semarang pada 21 Agustus 2019. DOI: https://dx.doi.org/10.13140/RG.2.2.13716.86400

Setiawan, Adib Rifqi. (2019, June 26). Peningkatan Literasi Saintifik melalui Pembelajaran Biologi Menggunakan Pendekatan Saintifik. Journal of Biology Education, 2 (1): 223-235. URL: http://journal.stainkudus.ac.id/index.php/jobe/article/view/5278

Setiawan, Adib Rifqi. (2019, March 23). Penerapan Pendekatan Saintifik dalam Pembelajaran Biologi sebagai Upaya Melatih Literasi Saintifik Siswa Sekolah Menengah. Disampaikan dalam Seminar Nasional Biologi 2019 Inovasi Penelitian dan Pembelajaran Biologi III (IP2B III) dengan tema "Peran Masyarakat dan Pemerintah Dalam Pengelolaan Sumber Daya Alam dan Lingkungan" di Fakultas Matematika dan Ilmu Pengetahuan Alam Universitas Negeri Surabaya pada 23 Maret 2019. DOI: https://dx.doi.org/10.13140/RG.2.2.19798.60484

Setiawan, Adib Rifqi. (2019, March 23). Penggunaan Naḍom MabāīīAsyroh dalam Pembelajaran Biologi untuk Meningkatkan Motivasi dan Hasil Belajar. Disampaikan dalam Seminar Nasional Biologi 2019 Inovasi Penelitian dan Pembelajaran Biologi III (IP2B III) dengan tema "Peran Masyarakat dan Pemerintah Dalam Pengelolaan Sumber Daya Alam dan Lingkungan" di Fakultas Matematika dan Ilmu Pengetahuan Alam Universitas Negeri Surabaya pada 23 Maret 2019. DOI: https://dx.doi.org/10.13140/RG.2.2.33220.37763

Setiawan, Adib Rifqi. (2019, March 23). Upaya Meningkatkan Motivasi Belajar dalam Pembelajaran Ilmu Pengetahuan Alam (IPA) Melalui Bacaan Populer. Disampaikan dalam Seminar Nasional Biologi 2019 Inovasi Penelitian dan Pembelajaran Biologi III (IP2B III) dengan tema "Peran Masyarakat dan Pemerintah Dalam Pengelolaan Sumber Daya Alam dan Lingkungan" di Fakultas Matematika dan Ilmu Pengetahuan Alam Universitas Negeri Surabaya pada 23 Maret 2019. DOI: https://dx.doi.org/10.13140/RG.2.2.13087.71847

Setiawan, Adib Rifqi. (2019, May 07). Penerapan Pendekatan Saintifik untuk Melatih Literasi Saintifik dalam Domain Kompetensi pada Topik Gerak Lurus di Sekolah Menengah Pertama. Prosiding Seminar Nasional Fisika (SiNaFi) 2018, 4 (1): 7-13. URL: http://proceedings.upi.edu/index.php/sinafi/article/view/355

Setiawan, Adib Rifqi. (2019, October 02). Efektivitas Pembelajaran Biologi Berorientasi Literasi Saintifik. Thabiea : Journal of Natural Science Teaching, 2 (2): 83-94. DOI: http://dx.doi.org/10.21043/thabiea.v2i2.5345

Setiawan, Adib Rifqi. (2019, September 11). Designing a Test of Scientific Literacy Competences for Elementary Education. EdArXiv. DOI: https://dx.doi.org/10.35542/osf.io/m8c5b

Setiawan, Adib Rifqi. (2019, September 28). Aligning Education for Sustainable Development (ESD) and Traditional Islamic Perspective. EdArXiv. DOI: https://dx.doi.org/10.35542/osf.io/gtsef 
Setiawan, Adib Rifqi. (2019, September 29). Constructing Thematic Learning Lesson Plan to Guide Primary Education Student on Achieving Scientific Literacy. EdArXiv. DOI: https://dx.doi.org/10.35542/osf.io/dn87g

Setiawan, Adib Rifqi. (2019, September 30). Instrumen Penilaian untuk Pembelajaran Ekologi Berorientasi Literasi Saintifik. Assimilation: Indonesian Journal of Biology Education (AIJBE), 2 (2): 42-46. DOI: https://dx.doi.org/10.17509/aijbe.v2i2.19250

Setiawan, Adib Rifqi; \& Koimah, Siti. (2019, September 23). Effective Learning and Teaching. Thesis Commons. DOI: https://dx.doi.org/10.31237/osf.io/p42nx

Setiawan, Adib Rifqi; \& Yulidarti, Sherly. (2019, July 10). Designing Worksheet to Guide Primary Education Student on Achieving Scientific Literacy. Thesis Commons. DOI: https://dx.doi.org/10.31237/osf.io/e27wq

Setiawan, Adib Rifqi; Utari, Setiya; Nugraha, Muhamad Gina. (2016, December 17). Mengonstruksi Rancangan Soal Domain Kompetensi Literasi Saintifik Siswa SMP Kelas VIII pada Topik Gerak Lurus. Disampaikan dalam Seminar Nasional Fisika (SiNaFi) 2016 dengan tema "Membangun Masyarakat Indonesia Berliterasi Sains dan Teknologi Abad 21 melalui Pendidikan dan Penelitian Fisika" di Departemen Pendidikan Fisika Universitas Pendidikan Indonesia (UPI) Bandung pada 17 Desember 2016. DOI: https://dx.doi.org/10.13140/RG.2.2.31856.10246/1

Setiawan, Adib Rifqi; Utari, Setiya; Nugraha, Muhamad Gina. (2017, 22 September). Mengonstruksi Rancangan Soal Domain Kompetensi Literasi Saintifik Siswa SMP Kelas VIII pada Topik Gerak Lurus. Jurnal WaPFi (Wahana Pendidikan Fisika), 2 (2), hlm. 44-48. DOI: http://dx.doi.org/10.17509/wapfi.v2i2.8277

Setiawan; Adib Rifqi. (2019, October 04). The Difference Between Hake's Normalized Gain g and Effect Size Cohen's d for Measuring the Improvement of Student's Scientific Literacy. EdArXiv. DOI: https://doi.org/10.35542/osf.io/fxu4t

Setiawan; Adib Rifqi; \& Van Bee, Jeffa Lianto. (2019, September 30). Ethnomathematics at a Glance. EdArXiv. DOI: https://dx.doi.org/10.35542/osf.io/mfsdc

Setiawan; Adib Rifqi; \& Van Bee, Jeffa Lianto. (2019, September 30). Ethnomathematics at a Glance. EdArXiv. DOI: https://dx.doi.org/10.35542/osf.io/mfsdc

Si'ayah, S., \& Setiawan, A. R. (2019). Constructing Learning Contents that Align with Traditional Islamic Perspective and Literacy Competencies.

Si'ayah, S., \& Setiawan, A. R. (2019). Multiple Intelligences Survey: Analysis on Validity and Reliability of Bahasa Indonesia Version Through Different Education Level.

Si'ayah, S., \& Setiawan, A. R. (2019). The Historical Trajectory of the Madrasah Banat Nahdlatul Ulama Kudus.

Si'ayah, S., Kurniawati, N. K., Velasufah, W., \& Setiawan, A. R. (2019). A Brief Explanation of Basic Science Education.

Si'ayah, Syarofis; Kurniawati, Novi Khoirunnisa; Velasufah, Whasfi; Setiawan, Adib Rifqi. (2019, September 30). A Brief Explanation of Basic Science Education. EdArXiv. DOI: https://dx.doi.org/10.35542/osf.io/z62w8

Siayah, S., Setiawan, A. R., \& Van Bee, J. L. (2019). The Impact of Inquiry Based Learning Implementation In Indonesia to Secondary School Student's Achievement.

Si'ayah, Syarofis, \& Setiawan, A. R. (2019, September 21). The Historical Trajectory of the Madrasah Banat Nahdlatul Ulama Kudus. Thesis Commons. DOI: https://dx.doi.org/10.31237/osf.io/qzpw9

Si'ayah, Syarofis, \& Setiawan, A. R. (2019, September 29). Multiple Intelligences Survey: Analysis on Validity and Reliability of Bahasa Indonesia Version Through Different Education Level. Thesis Commons. DOI: https://dx.doi.org/10.31237/osf.io/x2etf

Si'ayah, Syarofis, Kurniawati, Novi Khoirunnisa, Velasufah, Whasfi, \& Setiawan, A. R. (2019, September 30). A Brief Explanation of Basic Science Education. EdArXiv. DOI: https://dx.doi.org/10.35542/osf.io/z62w8

Si'ayah, Syarofis; \& Setiawan, Adib Rifqi. (2019, June 26). Multiple Intelligences Survey: Analysis on Validity and Reliability of Bahasa Indonesia Version Through Different Education Level. Thesis Commons. DOi; https://dx.doi.org/10.31237/osf.io/x2etf

Si'ayah, Syarofis; Setiawan, A. R., \& Van Bee, Jeffa Lianto. (2019, October 04). The Impact of Inquiry Based Learning Implementation In Indonesia to Secondary School Student's Achievement. Open Science Framework (OSF). DOI: https://doi.org/10.31219/osf.io/nad87

Velasufah, W., \& Setiawan, A. R. (2019). Analisis Potensi dan Arahan Strategi Kebijakan Pengembangan Desa Ekowisata di Wilayah Muria.

Velasufah, W., \& Setiawan, A. R. (2019). Erotic Capital at a Glance.

Velasufah, W., \& Setiawan, A. R. (2019). Memetakan Posisi Perempuan dalam Kitab Kuning. 
Velasufah, W., \& Setiawan, A. R. (2019). Menelusuri Kata Bermakna Manusia dalam al-Qur'ān. Velasufah, W., \& Setiawan, A. R. (2019). Mewujudkan Sistem Pendidikan Islam yang ProPerempuan.

Velasufah, W., \& Setiawan, A. R. (2019). Nilai Pesantren sebagai Dasar Pendidikan Karakter. Velasufah, W., \& Setiawan, A. R. (2019). Science Motivation Questionnaire II (SMQ-II): Analysis on Validity and Reliability of Bahasa Indonesia Version Through Various Learning Context.

Velasufah, W., \& Setiawan, A. R. (2019). The Development of Coffee Shopping Tourism in Colo.

Velasufah, W., \& Setiawan, A. R. (2019). The Value of the Persatuan Islam (PERSIS).

Velasufah, Whasfi, \& Setiawan, A. R. (2019, September 28). Analisis Potensi dan Arahan Strategi Kebijakan Pengembangan Desa Ekowisata di Wilayah Muria. Thesis Commons. DOI: https://dx.doi.org/10.31237/osf.io/st4qz

Velasufah, Whasfi, \& Setiawan, A. R. (2019, September 28). Memetakan Posisi Perempuan dalam Kitab Kuning. Thesis Commons. DOI: https://dx.doi.org/10.31237/osf.io/jq6uk

Velasufah, Whasfi, \& Setiawan, A. R. (2019, September 28). Menelusuri Kata Bermakna Manusia dalam al-Qur'ān. Thesis Commons. DOI: https://dx.doi.org/10.31237/osf.io/m5726

Velasufah, Whasfi, \& Setiawan, A. R. (2019, September 28). Mewujudkan Sistem Pendidikan Islam yang Pro-Perempuan. Thesis Commons. DOI: https://dx.doi.org/10.31237/osf.io/gs7c3

Velasufah, Whasfi, \& Setiawan, A. R. (2019, September 28). The Development of Coffee Shopping Tourism in Colo. Thesis Commons. DOI: https://dx.doi.org/10.31237/osf.io/yde8u

Velasufah, Whasfi, \& Setiawan, A. R. (2019, September 29). Science Motivation Questionnaire II (SMQ-II): Analysis on Validity and Reliability of Bahasa Indonesia Version Through Various Learning Context. Thesis Commons. DOI: https://dx.doi.org/10.31237/osf.io/xdjqt

Velasufah, Whasfi, \& Setiawan, A. R. (2019, September 30). Nilai Pesantren sebagai Dasar Pendidikan Karakter. EdArXiv. DOI: https://dx.doi.org/10.35542/osf.io/j9vtp

Velasufah, Whasfi; \& Setiawan, A. R. (2019, October 1). The Value of the Persatuan Islam (PERSIS). Thesis Commons. DOI: https://dx.doi.org/10.31237/osf.io/pvy7k

Velasufah, Whasfi; \& Setiawan, Adib Rifqi. (2019, August 09). Science Motivation Questionnaire II (SMQ-II): Analysis on Validity and Reliability of Bahasa Indonesia Version Through Various Learning Context. Thesis Commons. DOI: https://dx.doi.org/10.31237/osf.io/xdjqt

Yuliyahya, L., \& Setiawan, A. R. (2019). Pengaruh sifat matematika terhadap pembelajaran.

Zein, L. F., \& Setiawan, A. R. (2019). A Portal into Mémoires D'hadrien.

Zein, L. F., \& Setiawan, A. R. (2019). Apakah Orang Religius Kurang Toleran?.

Zein, L. F., \& Setiawan, A. R. (2019). Aperçu Général sur l'Écofeminisme.

Zein, L. F., \& Setiawan, A. R. (2019). Education During the Empire of Japan.

Zein, L. F., \& Setiawan, A. R. (2019). General Overview of Ecofeminism.

Zein, L. F., \& Setiawan, A. R. (2019). Historical Trajectory of Kitab Kuning.

Zein, L. F., \& Setiawan, A. R. (2019). Islamic Education Institutions in Singapore.

Zein, L. F., \& Setiawan, A. R. (2019). Julia Perez: une authentique modèle.

Zein, L. F., \& Setiawan, A. R. (2019). Kajian semiotika terhadap lagu julia perez.

Zein, L. F., \& Setiawan, A. R. (2019). Kecantikan Perempuan dalam As If It's Your Last karya BLACKPINK.

Zein, L. F., \& Setiawan, A. R. (2019). Kim Kardashian: the Leader of the Influencers.

Zein, L. F., \& Setiawan, A. R. (2019). Konsep Dasar Seksualitas.

Zein, L. F., \& Setiawan, A. R. (2019). La Valeur De La Capitale Érotique.

Zein, L. F., \& Setiawan, A. R. (2019). La Villa Isola.

Zein, L. F., \& Setiawan, A. R. (2019). Melihat Tempat Perempuan dalam kitab Kuning.

Zein, L. F., \& Setiawan, A. R. (2019). Pendidikan Karakter Berbasis Nilai Pesantren.

Zein, L. F., \& Setiawan, A. R. (2019). Salahkah Menjadi Perempuan Cantik?.

Zein, L. F., \& Setiawan, A. R. (2019). The Worth of Erotic Capital.

Zein, L. F., \& Setiawan, A. R. (2019). Women's Beauty in As If It's Your Last by BLACKPINK.

Zein, L. F., Safaningrum, A. C., \& Setiawan, A. R. (2019). Is 'God is a Woman'from Ariana Grande Only a Song? 